\title{
Assessment of Rock Mass Quality and Deformation Modulus by Empirical Methods along Kandiah River, KPK, Pakistan
}

\author{
Mian Sohail Akram, Kamran Mirza, Muhammad Zeeshan, Muhammad Asad Jabbar \\ Institute of Geology, University of the Punjab, Lahore, Pakistan \\ Email: sohail.geo@pu.edu.pk
}

How to cite this paper: Akram, M.S., Mirza, K., Zeeshan, M. and Jabbar, M.A. (2018) Assessment of Rock Mass Quality and Deformation Modulus by Empirical Methods along Kandiah River, KPK, Pakistan. Open Journal of Geology, 8, 947-964. https://doi.org/10.4236/ojg.2018.810057

Received: August 10, 2018

Accepted: September 18, 2018

Published: September 21, 2018

Copyright ( 92018 by authors and Scientific Research Publishing Inc. This work is licensed under the Creative Commons Attribution International License (CC BY 4.0). http://creativecommons.org/licenses/by/4.0/

\begin{abstract}
The pivotal aim of this study is to evaluate the rock mass characterization and deformation modulus. It is vital for rock mass classification to investigate important parameters of discontinuities. Therefore, Rock Mass Rating (RMR) and Tunneling quality index $(\mathrm{Q})$ classification systems are applied to analyze 22 segments along proposed tunnel routes for hydropower in Kandiah valley, Khyber Pakhtunkhwa, Pakistan. RMR revealed the range of fair to good quality rocks, whereas $\mathrm{Q}$ yielded poor to fair quality rocks for investigated segments of the rock mass. Besides, $E_{\mathrm{m}}$ values were acquired by empirical equations and computer-aided program RocLab, and both methods presented almost similar variation trend of their results. Hence, the correlations of $E_{\mathrm{m}}$ with $\mathrm{Q}$ and RMR were carried out with higher values of the regression coefficient. This study has scientific significance to initially understand the rock mass conditions of Kandiah valley.
\end{abstract}

\section{Keywords}

Rock Mass Classification, RMR and Q, Deformation Modulus $\left(E_{m}\right)$, Empirical Equations, RocLab, Tunnel

\section{Introduction}

Geomechanical investigation of the rock mass is an essential part of the feasibility phase of hydropower projects when very little information is available, to ascertain the response of rock behavior under disturbance or excavation. Rock mass characteristics are determined by empirical classification systems to classify the rock mass [1]. Hence, Rock Mass Rating (RMR) and Tunneling quality index (Q) classification systems are pivotal to classify the rock mass. Extensive studies 
have been conducted by using RMR and Q schemes e.g. [2]-[12]. Among the rock mass parameters, deformation modulus $\left(E_{\mathrm{m}}\right)$ has very significance in rock mechanics because it provides the initial idea about mechanical behavior of rock mass before failure. In this regard, there are several direct procedures to determine the deformation modulus in the laboratory, but these methods are costly and time consuming. Therefore, empirical methods have been suggested for indirect estimation of deformation modulus [13] [14]. As a consequence, various researchers [7] [15]-[25] have been proposed different empirical relations for estimation of deformation modulus.

Pakistan is facing a serious shortage of electricity, and Government is trying to develop hydropower, especially in Northern Pakistan to overcome the electricity disorder. In this regard, small hydropower is proposed along Kandiah River in Kandiah valley, KPK, Pakistan. Hence, the present study focuses on preliminary rock mass characterization with an assessment of required support and estimation of deformation modulus along proposed tunnel routes. Therefore, to achieve this goal, field observations including geological mapping, discontinuity surveys and sampling were conducted.

\section{Geological Setting of Study Area}

The study area is near about $30 \mathrm{Km}$ long V-shaped valley with steep slopes on either side of Kandiah River. Tectonically it is situated in Kohistan Island Arc (KIA) and surrounded by two sutures formed by the collision of Indian plate with Eurasian plate, whereas the first suture is known as northern suture from Eurasian plate and the second suture is between Main Mantel Thrust (MMT) and Indian plate, respectively (Figure 1) [26]. Chilas Complex (CC) and Gilgit Complex (GC) are two prevailing major geological units in the study area. The $\mathrm{CC}$ dominantly composed of intermediate to basic plutonic rocks such as diorite (Figure2(a)), gabbro, anorthosite, pyroxenite and GC comprising metasediments such as psammite (Figure2(b)) and protolith of metasediments. Moreover, Pleistocene unconsolidated deposits are also observed in the valley at different places, which are the result of river bed deposits, fan deposits, talus, fluvial deposits, scree and glacial deposits.

\section{Data and Methods}

\subsection{Data}

\section{Geological Mapping and Discontinuity Survey}

Tunnel routes were divided into segments and various traverses were made to mark geological contacts (Figure 3). According to International Society for Rock Mechanics [27], physical parameters (orientation, spacing, persistence, aperture, roughness, the number of joint sets, infilling material and hydraulic conditions)were frequently executed of all those discontinuities that were intersecting the reference line by measuring tape of approx. $10 \mathrm{~m}$ during scanline surveys [28]. 


\subsection{Methods}

\subsubsection{Rock Mass Rating (RMR) and Tunneling Quality Index (Q)}

RMR and Q are universal classification systems, and these systems have been applied by many researchers in tunneling and underground excavation. Bieniawski [29] developed RMR system by providing quantitative data for reinforcement of tunnel techniqueslike rock bolts, shotcrete etc. The modifications

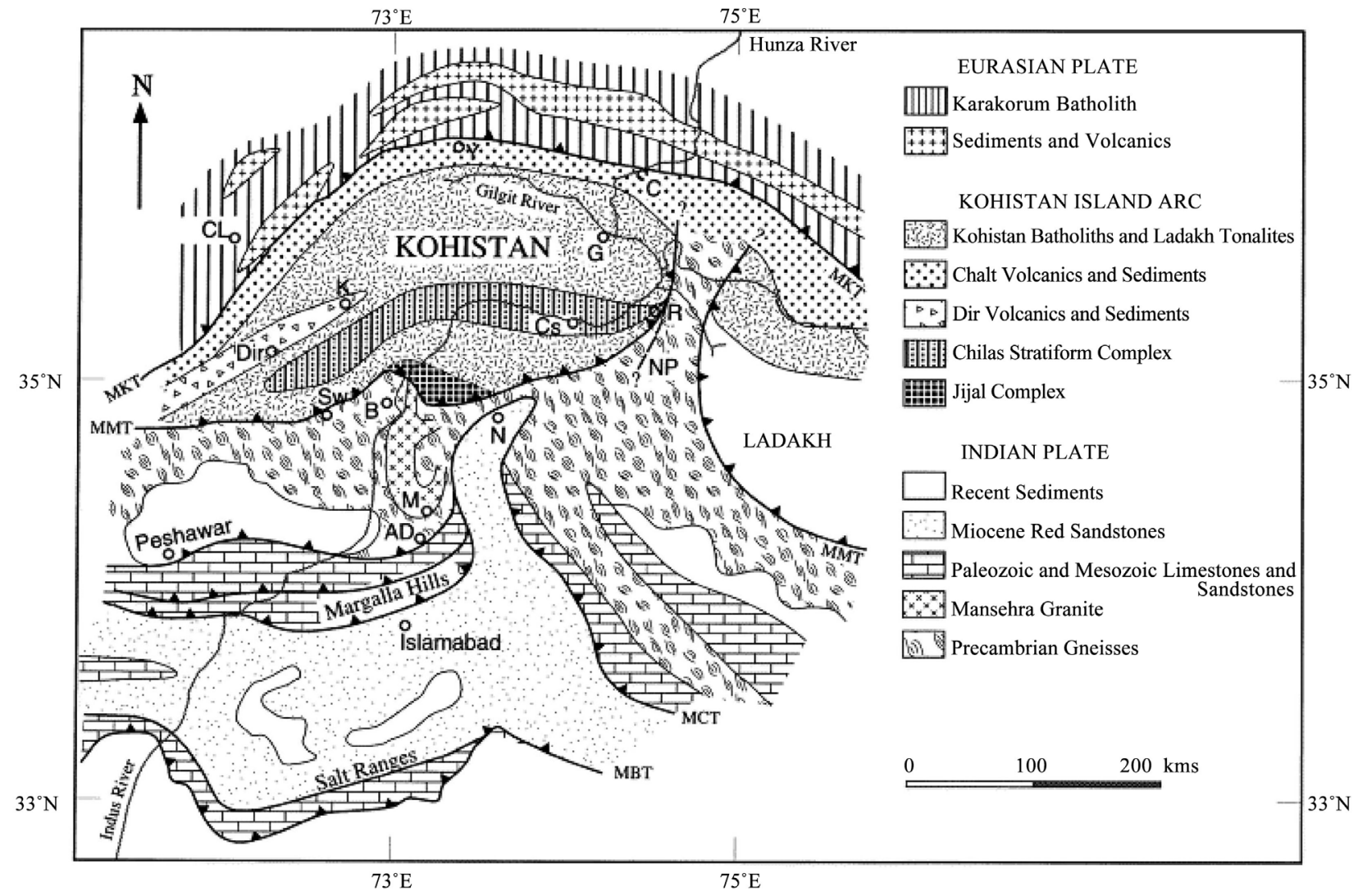

Figure 1. Regional tectonic map of study area (Modified after Tahirkheli and Jan [26]).
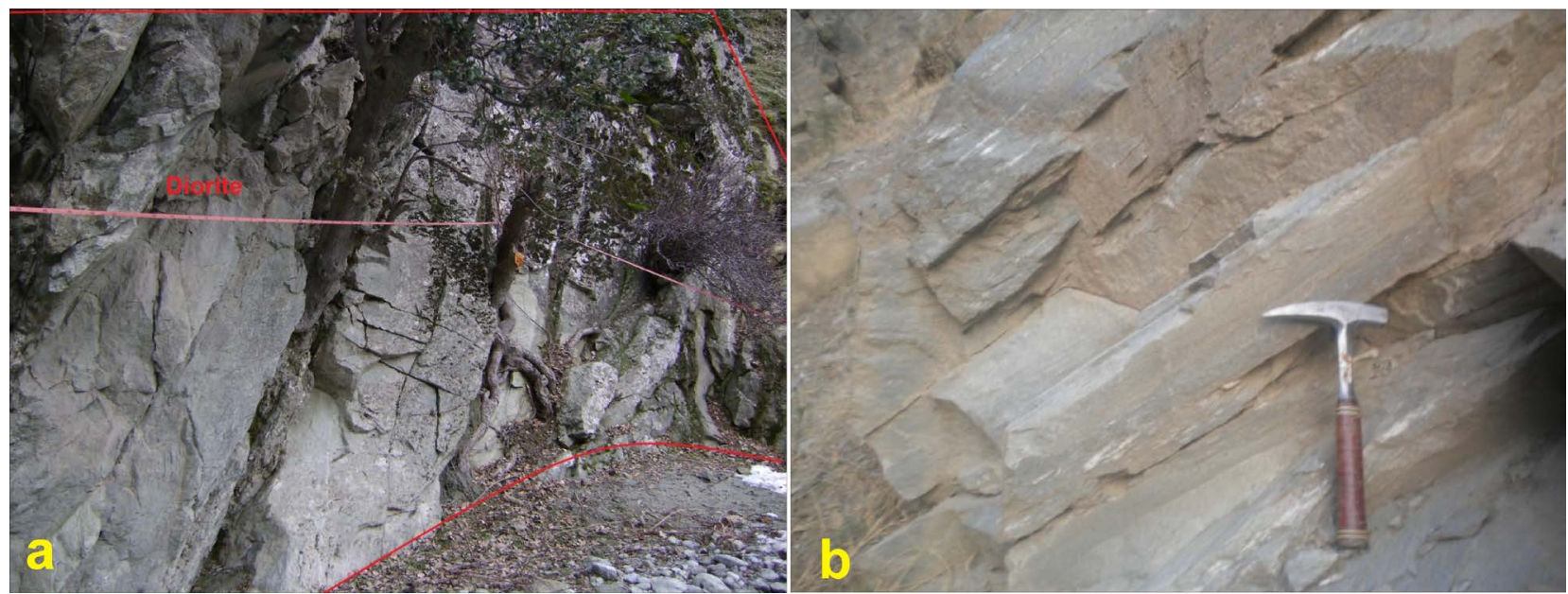

Figure 2. Exposed Rocks Unit of (a) Diorite belonging to Chilas Complex; (b) Psammite belonging to Gilgit Complex in study area. 


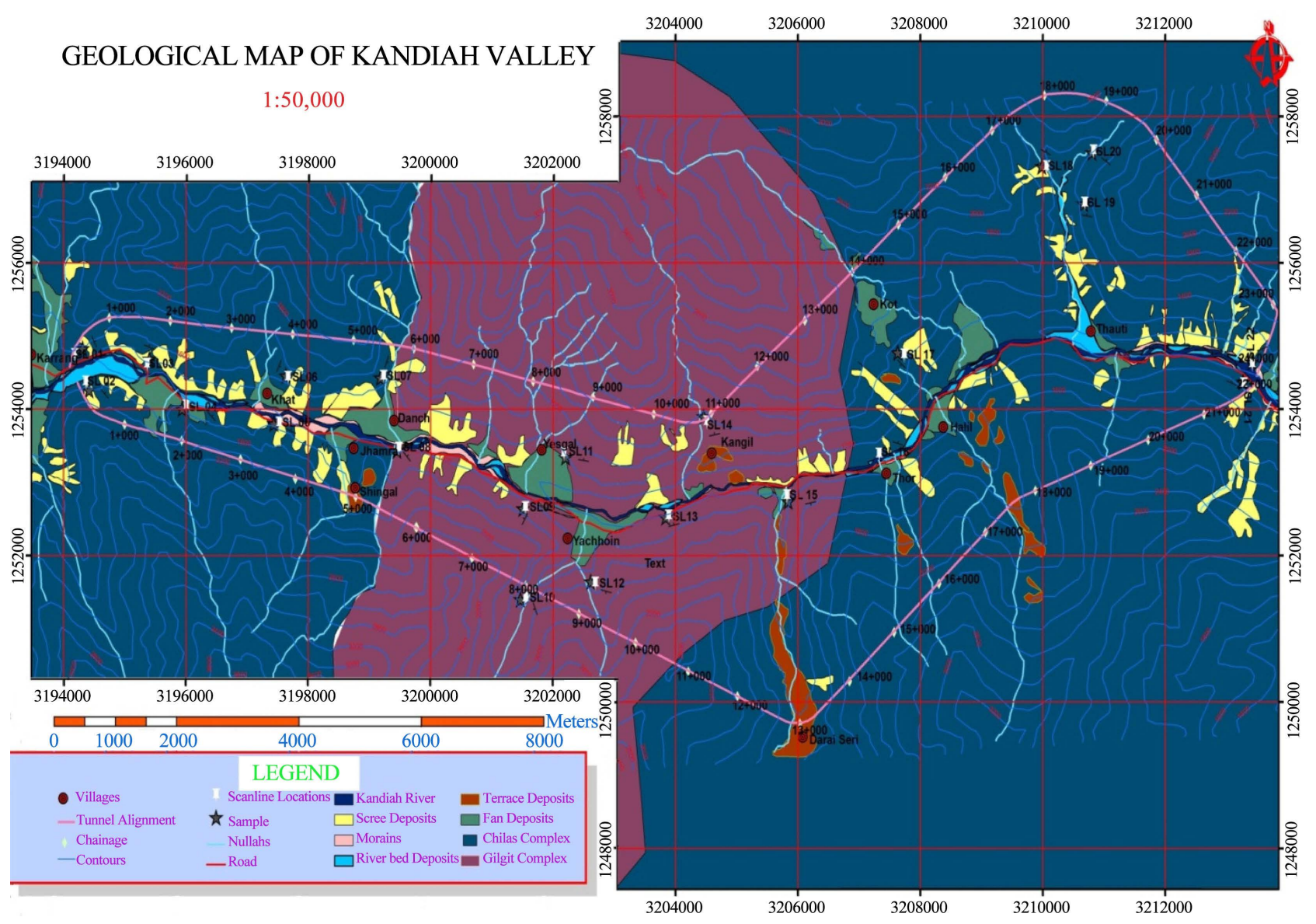

Figure 3. Geological map of study area.

had made over the many years e.g. [29] [30] [31] [32] [33]. In beginning, the system was established for only tunnels but with the passage of time, this system is also used for foundations, rock slopes, and mining problems. The following parameters were investigated during field work to calculate the RMR values: Uniaxial compressive strength (UCS), spacing, rock quality designation (RQD), ground water conditions, discontinuity conditions and orientation of discontinuities [3] [8] [10]. The RMR values are estimated by following equation [29]:

$$
R M R=R_{1}+R_{2}+R_{3}+R_{4}+R_{5}+R_{6}
$$

where, $R_{1}-R_{6}$ are the above mentioned parameters of discontinuities.

Barton et al. [34] proposed Q system that was used to determine rock mass quality and required support estimation for tunnels. The overall $\mathrm{Q}$ values ranged between 0.001 (exceptionally poor) to 1000 (exceptionally good) and can be estimated by using this expression:

$$
\mathrm{Q}=(\mathrm{RQD} / \mathrm{Jn}) \times(\mathrm{Jr} / \mathrm{Ja}) \times(\mathrm{Jw} / \mathrm{SRF})
$$

RQD is rock quality designation, $\mathrm{J}_{\mathrm{n}}$ is the joint set number, $\mathrm{J}_{\mathrm{r}}$ and $\mathrm{J}_{\mathrm{a}}$ are the ratings of roughness and alteration number, $J_{w}$ is for water inflow and pressure effects, and SRF is the stress reduction factor. Moreover, terms $\mathrm{Jr} / \mathrm{Ja}$ represents peak strength, $\mathrm{RQD} / \mathrm{Jn}$ indicates the relative block size and Jw/SRF related 
to the effective strength of the rock mass. It is noticed by the Equation (1) and Equation (2) that RMR values are calculated by summation of all assigned ratings, whereas $\mathrm{Q}$ values are calculated by divisions and products of assigned ratings to parameters.

\subsubsection{Estimation of Deformation Modulus Based on RMR and Q}

There are several equations proposed by different researchers to estimate deformation modulus $\left(E_{m}\right)$ based on Geomechanical classification systems e.g. [4] [15] [17] [18] [19] [20] [24] [35] [36] [37] [38] [39]. Most widely used equations by different researchers were plotted in Figure 4 by Hoek and Diederichs [7] and relevant equations are listed in Table 1.

In order to calculate the deformation modulus of rock mass, at least rating value of one rock mass classification system is required because joint's properties (e.g. roughness, weathering, infilling material, aperture, persistence, etc.) have significant effect on rock mass deformation [24] [40]. Hence, equations proposed by different researchers (Table 2) in which RMR and Q values considered as input parameters to estimate $E_{m}$.

\section{Results and Discussions}

\subsection{Rock Mass Classification}

This paper highlights the characterization of rock mass by RMR and Q schemes. Furthermore, discontinuity surveys were conducted at various locations to collect the required parameters for the estimation of RMR and $\mathrm{Q}$ values. The orientation data of discontinuities were analysed by computer program DIPS (version 5.1) that show mostly 2 to 3 joints sets were prevailing in the study area. The field surveys revealed that discontinuity's trend was mostly dipping towards the tunnel axis but at some points, the trend was away from tunnel axis, as well as at few locations strike was parallel to the tunnel axis.

The RMR values vary from 53 (fair) to 65 (good) with a mean of 57 (Table 3) on left route and values ranged between 51 (fair) to 62 (good) with average 56 (Table 4) for the right route of the tunnel. Moreover, $\mathrm{Q}$ values of rock mass ranged between 1.60 (poor) to 6.13 (fair) with an average of 3.22 along left tunnel route and 1.60 (poor) to 4.27 (fair) with an average of 2.81 along right tunnel alignment. The detail ratings of all required parameters with $\mathrm{Q}$ values are listed in Table 5 and Table 6.

The comparisons of RMR and Q values were analyzed by using the results of input parameters to calculate the empirical ratings for tunnel alignments. Along left tunnel route, RMR designated ten segments as a fair rock and only one segment $(20+000-22+000)$ show good rock but according to Q system, same segments were designated as a poor rock except for three segments $(3+000-5+000$, $17+000-19+000,20+000-22+000)$ that revealed a fair quality rock. Similarly, values of RMR along different segments of right tunnel route gave fair rock quality except for one segment $(12+000-15+000)$ that designated as good quali- 
ty of rock and Q system designated various segments as poor quality except for one segment $(15+000-20+000)$ that presented fair quality of rock mass. The calculated ratings suggest that $\mathrm{Q}$ system provided a more conservative approach as compare to RMR system for rock mass classification. The variation in values of RMR and Q plotted in Figure 5 and estimated support for specific rock class is summarized in Table 7.

Table 1. Empirical equations and field data of different researchers plotted in Figure 4 (after Hoek and Diederichs [7]).

\begin{tabular}{|c|c|c|c|}
\hline Plots and curved & Equations & Equation No. & Researcher (s) \\
\hline$\bigcirc$ & Field Data & & [17] \\
\hline$\diamond$ & Field Data & & [15] \\
\hline$\square$ & Field Data & & [35] \\
\hline 1 & $E_{m}=2 R M R-100$ for $R M R>50$ & 3 & [15] \\
\hline 2 & $E_{\mathrm{m}}=10^{(R M R-10) / 40}$ & 4 & {$[17]$} \\
\hline 3 & $E_{m}=E_{i} / 100\left(0.0028 R M R^{2}+0.9 \exp (R M R / 22.82)\right), E_{i}=50 \mathrm{Gpa}$ & 5 & [18] \\
\hline 4 & $E_{m}=E_{i}(0.5(1-\cos (\pi R M R / 100))), E_{i}=50 \mathrm{Gpa}$ & 6 & {$[19]$} \\
\hline 5 & $E_{m}=0.1(R M R / 10)^{3}$ & 7 & [20] \\
\hline 6 & $E_{\mathrm{m}}=10 Q_{c}^{1 / 3}$ where $Q_{c}=Q \sigma_{c i} / 100$ & 8 & {$[4]$} \\
\hline 7 & $E_{m}=\left(1-\frac{d}{2}\right) \sqrt{\frac{\sigma_{c i}}{100}} \times 10^{(R M R-10) / 40}$ & 9 & [38] \\
\hline 8 & $E_{m}=E_{i}\left(S^{a}\right)^{0.4} E_{i}=50 \mathrm{GPa}, s=\exp ((G S I-100) / 9)$ & 10 & {$[24]$} \\
\hline 9 & $E_{m}=E_{i} S^{1 / 4} E_{i}=50 \mathrm{GPa}, s=\exp ((G S I-100) / 9)$ & 11 & [37] \\
\hline 10 & $E_{m}=7( \pm 3) \sqrt{Q^{\prime}} Q^{\prime}=10((R M R-44) / 21)$ & 12 & [36] \\
\hline
\end{tabular}

Table 2. Empirical equations for estimation of deformation modulus $\left(E_{m}\right)$ by using RMR and Q values.

\begin{tabular}{cccc}
\hline Empirical Equation & Equation No. & Required Parameter & Reference \\
\hline$E_{m}(G P a)=40 \log Q(A v g)$. & 13 & $\mathrm{Q}$ & {$[41]$} \\
$E_{m}(G P a)=8 Q^{0.4}$ & 14 & $\mathrm{Q}$ & {$[21]$} \\
$E_{m}(G P a)=2 R M R-100$ for $R M R>50$ & 3 & $\mathrm{RMR}$ & {$[15]$} \\
$E_{m}(G P a)=0.1(R M R / 10)^{3}$ & 7 & $\mathrm{RMR}$ & {$[20]$} \\
$E_{m}(G P a)=5.6(R M R)^{0.375}$ & & $\mathrm{RMR}$ & {$[42]$} \\
$E_{m}(G P a)=0.0736 \mathrm{e}^{0.0755 R M R}$ & 15 & $\mathrm{RMR}$ & {$[22]$}
\end{tabular}


Table 3. Geomechanical classificationby RMR along left tunnel alignment (After [31]).

\begin{tabular}{|c|c|c|c|c|c|c|c|c|c|c|}
\hline \multicolumn{2}{|c|}{ Chainage } & \multirow{2}{*}{$\begin{array}{c}\text { Uniaxial } \\
\text { compressive } \\
\text { strength (Avg.) }\end{array}$} & \multirow{2}{*}{$\begin{array}{c}\text { Rock Quality } \\
\text { Designation } \\
\text { (Avg.) }\end{array}$} & \multirow{2}{*}{$\begin{array}{l}\text { Spacing } \\
\text { (Avg.) }\end{array}$} & \multirow{2}{*}{$\begin{array}{c}\text { Discontinuity } \\
\text { Condition }\end{array}$} & \multirow{2}{*}{$\begin{array}{c}\text { Water } \\
\text { Condition }\end{array}$} & \multirow{2}{*}{$\begin{array}{l}\text { RMR } \\
\text { Value }\end{array}$} & \multirow{2}{*}{ Description } & \multirow{2}{*}{$\begin{array}{l}\text { Rock } \\
\text { Class }\end{array}$} & \multirow{2}{*}{$\begin{array}{c}\text { GSI } \\
(\mathrm{RMR}-5)\end{array}$} \\
\hline From & To & & & & & & & & & \\
\hline $0+00$ & $1+000$ & 7 & 8 & 10 & & & 57 & Fair & III & 52 \\
\hline $1+000$ & $3+000$ & 12 & 8 & 8 & & & 56 & Fair & III & 51 \\
\hline $3+000$ & $5+000$ & 12 & 13 & 10 & Persistence > & & 59 & Fair & III & 54 \\
\hline $5+000$ & $7+000$ & 7 & 13 & 10 & $10-15 \mathrm{~m}$ & & 55 & Fair & III & 50 \\
\hline $7+000$ & $10+000$ & 7 & 17 & 10 & $\begin{array}{c}\text { aperture }> \\
1-5 \mathrm{~mm},\end{array}$ & $\begin{array}{l}\text { Approx. } \\
\text { Damp }\end{array}$ & 58 & Fair & III & 53 \\
\hline $10+000$ & $14+000$ & 7 & 13 & 10 & slightly rough & to & 57 & Fair & III & 52 \\
\hline $14+000$ & $17+000$ & 7 & 13 & 5 & $\begin{array}{l}\text { to rough, } \\
\text { slightly to }\end{array}$ & $\begin{array}{c}\text { completely } \\
\text { dry }\end{array}$ & 53 & Fair & III & 48 \\
\hline $17+000$ & $19+000$ & 12 & 8 & 15 & moderately & & 59 & Fair & III & 54 \\
\hline $19+000$ & $20+000$ & 7 & 8 & 15 & weathered & & 57 & Fair & III & 52 \\
\hline $20+000$ & $22+000$ & 7 & 13 & 15 & & & 65 & Good & II & 60 \\
\hline $22+000$ & $24+000$ & 7 & 13 & 15 & & & 57 & Fair & III & 52 \\
\hline
\end{tabular}

Table 4. Geomechanical classification by RMR along right tunnel alignment (After [31]).

\begin{tabular}{|c|c|c|c|c|c|c|c|c|c|c|}
\hline \multicolumn{2}{|c|}{ Chainage } & \multirow{2}{*}{$\begin{array}{c}\text { Uniaxial } \\
\text { compressive } \\
\text { strength (Avg.) }\end{array}$} & \multirow{2}{*}{$\begin{array}{c}\text { Rock Quality } \\
\text { Designation } \\
\text { (Avg.) }\end{array}$} & \multirow{2}{*}{$\begin{array}{l}\text { Spacing } \\
\text { (Avg.) }\end{array}$} & \multirow{2}{*}{$\begin{array}{l}\text { Discontinuity } \\
\text { Condition }\end{array}$} & \multirow{2}{*}{$\begin{array}{c}\text { Water } \\
\text { Condition }\end{array}$} & \multirow{2}{*}{$\begin{array}{l}\text { RMR } \\
\text { Value }\end{array}$} & \multirow{2}{*}{ Description } & \multirow{2}{*}{$\begin{array}{l}\text { Rock } \\
\text { Class }\end{array}$} & \multirow{2}{*}{$\begin{array}{c}\text { GSI } \\
(\mathrm{RMR}-5)\end{array}$} \\
\hline From & To & & & & & & & & & \\
\hline $0+00$ & $2+000$ & 7 & 13 & 10 & \multirow{11}{*}{$\begin{array}{l}\text { Persistence }> \\
10-15 \mathrm{~m}, \\
\text { aperture }>15 \mathrm{~mm} \text {, } \\
\text { slightly rough to } \\
\text { rough, slightly to } \\
\text { moderately } \\
\text { weathered }\end{array}$} & \multirow{11}{*}{$\begin{array}{l}\text { Approx. } \\
\text { damp to } \\
\text { completely } \\
\text { dry }\end{array}$} & 59 & Fair & III & 54 \\
\hline $2+000$ & $3+000$ & 7 & 13 & 10 & & & 58 & Fair & III & 53 \\
\hline $3+000$ & $5+000$ & 7 & 8 & 10 & & & 51 & Fair & III & 46 \\
\hline $5+000$ & $6+000$ & 7 & 13 & 10 & & & 58 & Fair & III & 53 \\
\hline $6+000$ & $8+000$ & 7 & 8 & 10 & & & 55 & Fair & III & 50 \\
\hline $8+000$ & $9+000$ & 7 & 17 & 10 & & & 53 & Fair & III & 48 \\
\hline $9+000$ & $10+000$ & 12 & 13 & 10 & & & 53 & Fair & III & 48 \\
\hline $10+000$ & $12+000$ & 7 & 13 & 10 & & & 57 & Fair & III & 52 \\
\hline $12+000$ & $15+000$ & 12 & 13 & 10 & & & 62 & Good & II & 57 \\
\hline $15+000$ & $20+000$ & 7 & 13 & 8 & & & 57 & Fair & III & 52 \\
\hline $20+000$ & $22+000$ & 4 & 13 & 15 & & & 51 & Fair & III & 46 \\
\hline
\end{tabular}

Table 5. Estimated Q values along left tunnel alignment of KandiahRiver (After [34]).

\begin{tabular}{|c|c|c|c|c|c|c|c|c|c|}
\hline \multicolumn{2}{|c|}{ Chainage } & \multirow{2}{*}{-RQD (Avg.) } & \multirow{2}{*}{$\mathrm{J}_{\mathrm{N}}$} & \multirow{2}{*}{$\begin{array}{c}\mathrm{J}_{\mathrm{R}} \\
\text { (Avg.) }\end{array}$} & \multirow{2}{*}{$\mathrm{J}_{\mathrm{A}}$ (Avg.) } & \multirow{2}{*}{$\mathrm{J}_{\mathrm{w}}$ (Avg.) } & \multirow{2}{*}{ SRF (Avg.) } & \multirow{2}{*}{ Q-Value } & \multirow{2}{*}{ Descriptior } \\
\hline From & To & & & & & & & & \\
\hline $0+00$ & $1+000$ & 48 & 9 & 1.5 & 2 & 1 & 2.5 & 1.60 & Poor \\
\hline $1+000$ & $3+000$ & 50 & 9 & 3 & 2 & 1 & 2.5 & 3.33 & Poor \\
\hline $3+000$ & $5+000$ & 64 & 9 & 1.5 & 1 & 1 & 2.5 & 4.27 & Fair \\
\hline $5+000$ & $7+000$ & 64 & 9 & 1.5 & 2 & 1 & 2.5 & 2.13 & Poor \\
\hline $7+000$ & $10+000$ & 76 & 9 & 1.5 & 2 & 1 & 2.5 & 2.53 & Poor \\
\hline $10+000$ & $14+000$ & 60 & 9 & 1.5 & 2 & 1 & 2.5 & 2.00 & Poor \\
\hline $14+000$ & $17+000$ & 59 & 9 & 1.5 & 1 & 1 & 2.5 & 3.93 & Poor \\
\hline
\end{tabular}




\section{Continued}

\begin{tabular}{|c|c|c|c|c|c|c|c|c|c|}
\hline $17+000$ & $19+000$ & 46 & 9 & 3 & 1 & 1 & 2.5 & 6.13 & Fair \\
\hline $19+000$ & $20+000$ & 48 & 9 & 3 & 2 & 1 & 2.5 & 3.20 & Poor \\
\hline $20+000$ & $22+000$ & 62 & 9 & 3 & 2 & 1 & 2.5 & 4.13 & Fair \\
\hline $22+000$ & $24+000$ & 64 & 9 & 1.5 & 2 & 1 & 2.5 & 2.13 & Poor \\
\hline
\end{tabular}

Table 6. Estimated Q values along right tunnel alignment of KandiahRiver (After [34]).

\begin{tabular}{|c|c|c|c|c|c|c|c|c|c|}
\hline \multicolumn{2}{|c|}{ Chainage } & \multirow{2}{*}{-RQD (Avg.) } & \multirow{2}{*}{$\mathrm{J}_{\mathrm{N}}$} & \multirow{2}{*}{$\begin{array}{c}\mathrm{J}_{\mathrm{R}} \\
\text { (Avg.) }\end{array}$} & \multirow{2}{*}{$\mathrm{J}_{\mathrm{A}}$ (Avg.) } & \multirow{2}{*}{$\mathrm{J}_{\mathrm{W}}$ (Avg.) } & \multirow{2}{*}{ SRF (Avg.) } & \multirow{2}{*}{ Q-Value } & \multirow{2}{*}{ Description } \\
\hline From & To & & & & & & & & \\
\hline $0+00$ & $2+000$ & 56 & 9 & 3 & 2 & 1 & 2.5 & 3.73 & Poor \\
\hline $2+000$ & $3+000$ & 58 & 9 & 1.5 & 2 & 1 & 2.5 & 1.93 & Poor \\
\hline $3+000$ & $5+000$ & 48 & 9 & 1.5 & 2 & 1 & 2.5 & 1.60 & Poor \\
\hline $5+000$ & $6+000$ & 54 & 9 & 1.5 & 2 & 1 & 2.5 & 1.80 & Poor \\
\hline $6+000$ & $8+000$ & 48 & 9 & 1.5 & 1 & 1 & 2.5 & 3.20 & Poor \\
\hline $8+000$ & $9+000$ & 76 & 9 & 1.5 & 2 & 1 & 2.5 & 2.53 & Poor \\
\hline $9+000$ & $10+000$ & 56 & 9 & 3 & 2 & 1 & 2.5 & 3.73 & Poor \\
\hline $10+000$ & $12+000$ & 60 & 9 & 1.5 & 2 & 1 & 2.5 & 2.00 & Poor \\
\hline $12+000$ & $15+000$ & 68 & 9 & 1.5 & 2 & 1 & 2.5 & 2.27 & Poor \\
\hline $15+000$ & $20+000$ & 64 & 9 & 1.5 & 1 & 1 & 2.5 & 4.27 & Fair \\
\hline $20+000$ & $22+000$ & 58 & 9 & 3 & 2 & 1 & 2.5 & 3.87 & Poor \\
\hline
\end{tabular}

Table 7. Estimated support categories of rock mass according to RMR and Q (after [29] [41]).

\begin{tabular}{ccccc}
\hline Sr. No. & RMR values & Q values & Estimated support by RMR & Estimated support by Q \\
\hline 1 & $41-60$ & $1-4$ & $\begin{array}{c}\text { Systematic bolts } 4.0 \mathrm{~m} \text { long, } 1.5-2.0 \mathrm{~m} \text { spaced in crown } \\
\text { and walls with wire mesh in the crown. } 50-100 \mathrm{~mm} \\
\text { shotcrete in crown and } 30 \mathrm{~mm} .\end{array}$ & $\begin{array}{c}\text { Systematic bolting with } 40-100 \mathrm{~mm} \\
\text { unreinforced shotcrete. }\end{array}$ \\
2 & $61-80$ & $4-10$ & $\begin{array}{c}\text { Locally, bolts in crown } 3 \mathrm{~m} \text { long, spaced } 2.5 \mathrm{~m} \text { with occasional } \\
\text { wire mesh. Shotcrete } 50 \mathrm{~mm} \text { in crown where required. }\end{array}$ & Systematic bolting \\
\hline
\end{tabular}

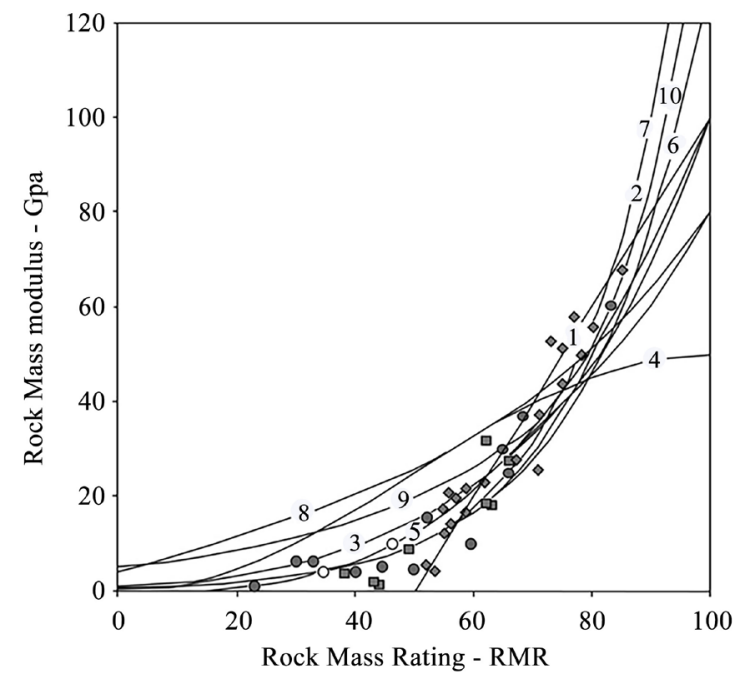

Figure 4. Comparison of rock mass deformation modulus by using different empirical equations with data from in situ measurements (after Hoek and Diederichs [7]). 

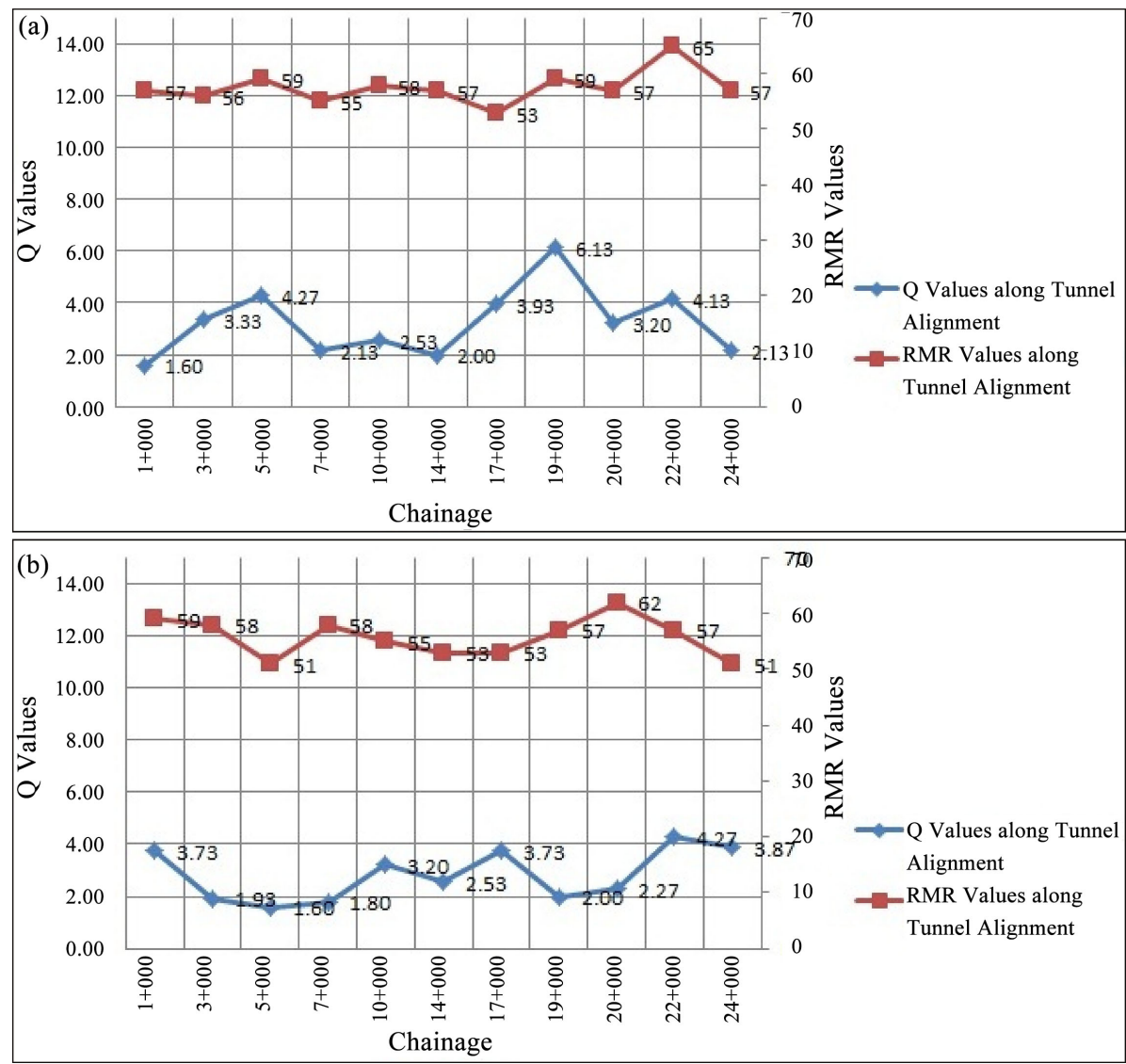

Figure 5. Comparison of RMR and Q values for (a) left tunnel alignment (b) right tunnel alignment.

\subsection{Estimation of Rock Mass Deformation Modulus}

In this study, $E_{\mathrm{m}}$ values were calculated for total 22 segments along tunnel alignments by widely accepted empirical equations and presented in Table 8 and Table 9. $E_{\mathrm{m}}$ values of left tunnel alignment obtained from Palmstrom and Singh [21] (9.65 - 16.53 GPa, Avg. 12.54 GPa) are on the lower side as compare to Grimstad and Barton [41] ranged between 8.16 - $31.51 \mathrm{GPa}$ with an average of $19 \mathrm{GPa}$ by using Q values. However, the range of $E_{\mathrm{m}}$ values calculated with RMR values by Bieniawski [15] is 6 - $30 \mathrm{GPa}$ with average $15.09 \mathrm{GPa}$ and by Read et al. [20] values range between 14.89 - 27.46 GPa (average 19.20 GPa). Similarly, $E_{\mathrm{m}}$ values vary between $4.02-9.96 \mathrm{GPa}$ with an average of $5.82 \mathrm{GPa}$ by Gokceoglu et al. [22] and the equation of Palmstrom [42] provides the values range of 24.82 $26.79 \mathrm{GPa}$ with average $25.59 \mathrm{GPa}$. The $E_{\mathrm{m}}$ values of right tunnel alignment calculated from Palmstrom and Singh [21] vary within the range of 9.65 - 14.29 GPa with an average of $11.94 \mathrm{GPa}$ and values by Grimstad and Barton [41] ranged between 8.16 - 25.20 GPa with an average of $16.99 \mathrm{GPa}$ by using Q values. Likewise, calculated values of $E_{\mathrm{m}}$ using RMR values by Bieniawski [15] vary between 2 - $24 \mathrm{GPa}$ with average $11.64 \mathrm{GPa}$ and by Read et al. [20] values range between 13.27 - 23.83 GPa with average 17.58. Likewise, $E_{\mathrm{m}}$ values vary between 3.46 - 7.94 GPa with average $5.14 \mathrm{GPa}$ by Gokceoglu et al. [22], and values cal- 
culated by Palmstrom [42] vary between 24.46 - 26.32 GPa with average 25.30 GPa.

The calculated values of $E_{\mathrm{m}}$ from empirical equations were compared with rock mass quality (Figure 6 and Figure 7) to understand the similarity or inconsistency emanating. However, the pattern of $E_{\mathrm{m}}$ with rock mass quality was observed significantly for all equations. According to Kayabasi et al. [23] and Panthee et al. [43], significant results between $E_{\mathrm{m}}$ calculated by using empirical equations and rock mass class were not observed but Hoek and Diederichs [7] revealed that $E_{\mathrm{m}}$ values increases with an increase in rock mass class (Figure 4) by using exponentially or power function (Table 1 ). In this study, $E_{\mathrm{m}}$ values were calculated by different equations where few equations gave high values, and several equations provided $E_{\mathrm{m}}$ values of the lower range.

The relationships of $E_{\mathrm{m}}$ with $\mathrm{Q}$ and RMR were derived and presented in Figure 8 and Figure 9 . The $E_{\mathrm{m}}$ values show significant positive correlation with $\mathrm{Q}$ and RMR. The $E_{\mathrm{m}}$ values for left tunnel alignment calculated by Grimstad and Barton [41] \& Palmstrom and Singh [21] show apositive correlation with Q values $\left(R^{2}=0.96\right.$ and 0.98$)$ as shown in Figure 8. Likewise, the calculated values of $E_{\mathrm{m}}$ with equations suggested by Bieniawski [15], Read et al. [20], Gokceoglu et al. [22], Palmstrom [42] have been plotted against RMR values (Figure 9) that presented direct correlation $\left(R^{2}=1,0.99,0.97\right.$ and 0.99). Similarly, $E_{\mathrm{m}}$ values for right tunnel alignment calculated by Grimstad and Barton [41] \& Palmstrom and Singh [21] provides positive correlation with $\mathrm{Q}$ values $\left(R^{2}=0.98\right.$ and 0.99$)$ as shown in Figure 8 and likewise, $E_{\mathrm{m}}$ values obtained from Bieniawski [15], Read et al. [20], Gokceoglu et al. [22], Palmstrom [42] were plotted against RMR values of right tunnel alignment and displays significant positive direct relationship ( $R^{2}=1,0.99,0.98$ and 0.99$)$ (Figure 9). In the light of above discussion, relations provided in Table 10 can be used to predict $E_{\mathrm{m}}$ by Q and RMR values with an accuracy of $R^{2}=0.96-1.00$ for similar properties of the rock mass and rock type of this study.

The $E_{\mathrm{m}}$ values were also determined by the computer-aided program RocLab by using various required parameters of rock mass like Geological strength index (GSI), UCS, etc. and listed in Table 8 and Table 9. Estimated $E_{\mathrm{m}}$ values for left tunnel alignment vary between 7.76 - 16.22 GPaby RocLab and $E_{\mathrm{m}}$ values (Avg.) of each segment acquired by all stated empirical equations ranged between 13.40 - 22.38 GPa. Similarly, for right tunnel alignment, RocLab provides the $E_{\mathrm{m}}$ values range of 7.14 - $14.99 \mathrm{GPa}$ and $E_{\mathrm{m}}$ values (Avg.) by all empirical equations along each segment ranged between 10.23 - $18.19 \mathrm{GPa}$. It can be observed in Figure 10 that fluctuation of obtained $E_{\mathrm{m}}$ values by both ways has the more or less similar trend. On the other hand, it should be noted that provided values by RocLab are on the lower side as compare to average values obtained by empirical equations (Figure 10). However, the present research is based on the data of few locations devoid of detail observation of rock mass. Therefore, results can be improved by conducting more discontinuity data in detail by following the same approach. 
Table 8. Calculated values of deformation modulus along left tunnel alignment.

\begin{tabular}{|c|c|c|c|c|c|c|c|c|}
\hline & & \multicolumn{2}{|c|}{$E_{\mathrm{m}}$ by using $\mathrm{Q}$ values } & \multicolumn{4}{|c|}{$E_{\mathrm{m}}$ by using $\mathrm{RMR}$ values } & \multirow{2}{*}{$\begin{array}{l}E_{\mathrm{m}} \text { by Using } \\
\text { RocLab GPa }\end{array}$} \\
\hline \multicolumn{2}{|c|}{ Chainage } & $\begin{array}{c}\text { Grimstad and } \\
\text { Barton [41] GPa }\end{array}$ & $\begin{array}{l}\text { Palmstrom and } \\
\text { Singh [21] GPa }\end{array}$ & $\begin{array}{c}\text { Bieniawski } \\
{[15] \mathrm{GPa}}\end{array}$ & $\begin{array}{l}\text { Read et al. } \\
{[20] \mathrm{GPa}}\end{array}$ & $\begin{array}{l}\text { Palmstrom } \\
\text { [42] GPa }\end{array}$ & $\begin{array}{c}\text { Gokceoglu et al. } \\
\text { [22] GPa }\end{array}$ & \\
\hline & & (Equation (13)) & (Equation (14)) & (Equation (3) & Equation (7) & Equation (15 & ) (Equation (16)) & \\
\hline $0+00$ & $1+000$ & 8.16 & 9.65 & 14.00 & 18.52 & 25.51 & 5.44 & 10.68 \\
\hline $1+000$ & $3+000$ & 20.92 & 12.95 & 12.00 & 17.56 & 25.34 & 5.05 & 11.66 \\
\hline $3+000$ & $5+000$ & 25.20 & 14.29 & 18.00 & 20.54 & 25.84 & 6.33 & 13.20 \\
\hline $5+000$ & $7+000$ & 13.16 & 10.83 & 10.00 & 16.64 & 25.17 & 4.68 & 9.18 \\
\hline $7+000$ & $10+000$ & 16.15 & 11.60 & 16.00 & 19.51 & 25.67 & 5.87 & 13.84 \\
\hline $10+000$ & $14+000$ & 12.04 & 10.56 & 14.00 & 18.52 & 25.51 & 5.44 & 12.14 \\
\hline $14+000$ & $17+000$ & 23.79 & 13.84 & 6.00 & 14.89 & 24.82 & 4.02 & 7.76 \\
\hline $17+000$ & $19+000$ & 31.51 & 16.53 & 18.00 & 20.54 & 25.84 & 6.33 & 14.08 \\
\hline $19+000$ & $20+000$ & 20.21 & 12.74 & 14.00 & 18.52 & 25.51 & 5.44 & 10.34 \\
\hline $20+000$ & $22+000$ & 24.65 & 14.11 & 30.00 & 27.46 & 26.79 & 9.96 & 16.22 \\
\hline $22+000$ & $24+000$ & 13.16 & 10.83 & 14.00 & 18.52 & 25.51 & 5.44 & 10.11 \\
\hline
\end{tabular}

Table 9. Calculated values of deformation modulus along right tunnel alignment.

\begin{tabular}{|c|c|c|c|c|c|c|c|c|}
\hline & & \multicolumn{2}{|c|}{$E_{\mathrm{m}}$ by using $\mathrm{Q}$ values } & \multicolumn{4}{|c|}{$E_{\mathrm{m}}$ by using RMR values } & \multirow{3}{*}{$\begin{array}{l}E_{\mathrm{m}} \text { by Using } \\
\text { RocLab GPa } \\
-\end{array}$} \\
\hline \multicolumn{2}{|c|}{ Chainage } & \multirow{2}{*}{$\begin{array}{c}\begin{array}{c}\text { Grimstad and } \\
\text { Barton [41] GPa }\end{array} \\
\text { (Equation (13)) }\end{array}$} & \multirow{2}{*}{$\begin{array}{l}\text { Palmstrom and } \\
\text { Singh [21] GPa } \\
\text { (Equation (14)) }\end{array}$} & \multirow{2}{*}{$\begin{array}{c}\begin{array}{c}\text { Bieniawski } \\
{[15] \mathrm{GPa}}\end{array} \\
\text { (Equation (3)) }\end{array}$} & \multirow{2}{*}{$\begin{array}{l}\text { Read et al. } \\
{[20] \mathrm{GPa}}\end{array}$} & \multirow{2}{*}{$\begin{array}{c}\text { Palmstrom } \\
{[42] \mathrm{GPa}}\end{array}$} & \multirow{2}{*}{$\begin{array}{c}\begin{array}{c}\text { Gokceoglu } \\
\text { et al. [22] GPa }\end{array} \\
\text { (Equation (16)) }\end{array}$} & \\
\hline & & & & & & & & \\
\hline $0+00$ & $2+000$ & 22.88 & 13.55 & 18.00 & 20.54 & 25.84 & 6.33 & 11.32 \\
\hline $2+000$ & $3+000$ & 11.45 & 10.41 & 16.00 & 19.51 & 25.67 & 5.87 & 11.42 \\
\hline $3+000$ & $5+000$ & 8.16 & 9.65 & 2.00 & 13.27 & 24.46 & 3.46 & 7.14 \\
\hline $5+000$ & $6+000$ & 10.21 & 10.12 & 16.00 & 19.51 & 25.67 & 5.87 & 13.34 \\
\hline $6+000$ & $8+000$ & 20.21 & 12.74 & 10.00 & 16.64 & 25.17 & 4.68 & 10.78 \\
\hline $8+000$ & $9+000$ & 16.15 & 11.60 & 6.00 & 14.89 & 24.82 & 4.02 & 10.26 \\
\hline $9+000$ & $10+000$ & 22.88 & 13.55 & 6.00 & 14.89 & 24.82 & 4.02 & 10.63 \\
\hline $10+000$ & $12+000$ & 12.04 & 10.56 & 14.00 & 18.52 & 25.51 & 5.44 & 12.14 \\
\hline $12+000$ & $15+000$ & 14.22 & 11.10 & 24.00 & 23.83 & 26.32 & 7.94 & 14.99 \\
\hline $15+000$ & $20+000$ & 25.20 & 14.29 & 14.00 & 18.52 & 25.51 & 5.44 & 9.89 \\
\hline $20+000$ & $22+000$ & 23.49 & 13.74 & 2.00 & 13.27 & 24.46 & 3.46 & 7.30 \\
\hline
\end{tabular}


Table 10. Correlations of $E_{m}$ with RMR and Q values for present study area.

\begin{tabular}{cccc}
\hline Parameters & Relation & $R^{2}$ & Equation No. \\
\hline \multirow{2}{*}{$\mathrm{Q}$} & $E_{\mathrm{m}}=6.3036 \mathrm{Q}-0.7354$ & 0.98 & 16 \\
$E_{\mathrm{m}}=1.7308 \mathrm{Q}+7.071$ & 0.99 & 17 \\
$E_{\mathrm{m}}=5.1659 \mathrm{Q}+2.371$ & 0.96 & 18 \\
$E_{\mathrm{m}}=1.5264 \mathrm{Q}+7.6272$ & 0.98 & 19 \\
$E_{\mathrm{m}}=2 \mathrm{RMR}-100$ & 1.00 & 20 \\
& $E_{\mathrm{m}}=1.0556 \mathrm{RMR}-41.541$ & 0.99 & 21 \\
$\mathrm{RMR}$ & $E_{\mathrm{m}}=0.5008 \mathrm{xRMR}-22.998$ & 0.97 & 22 \\
& $E_{\mathrm{m}}=0.1641 \mathrm{RMR}+16.148$ & 0.99 & 23 \\
$E_{\mathrm{m}}=0.9402 \mathrm{RMR}-34.899$ & 0.99 & 24 \\
$E_{\mathrm{m}}=0.387 \mathrm{RMR}-16.461$ & 0.98 & 25 \\
$E_{\mathrm{m}}=0.17 \mathrm{RMR}+15.804$ & 0.99 & 26 \\
\hline
\end{tabular}

${ }^{*} \mathrm{y}$ is for $E_{\mathrm{m}}$ and $\mathrm{x}$ for RMR \& $\mathrm{Q}$.

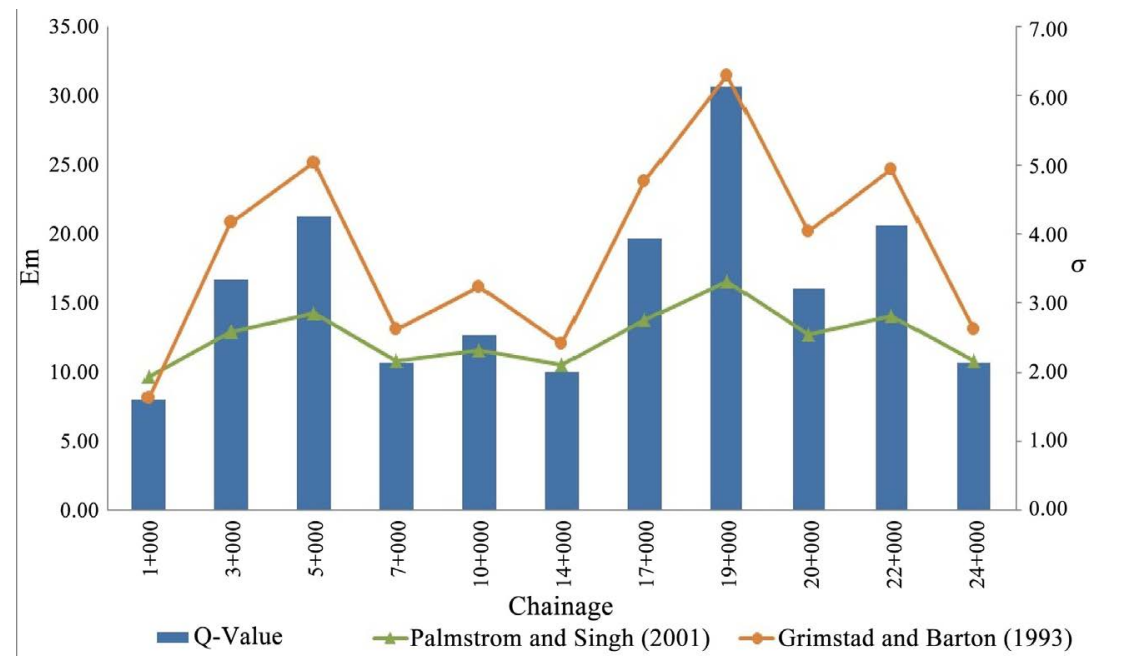

(a)

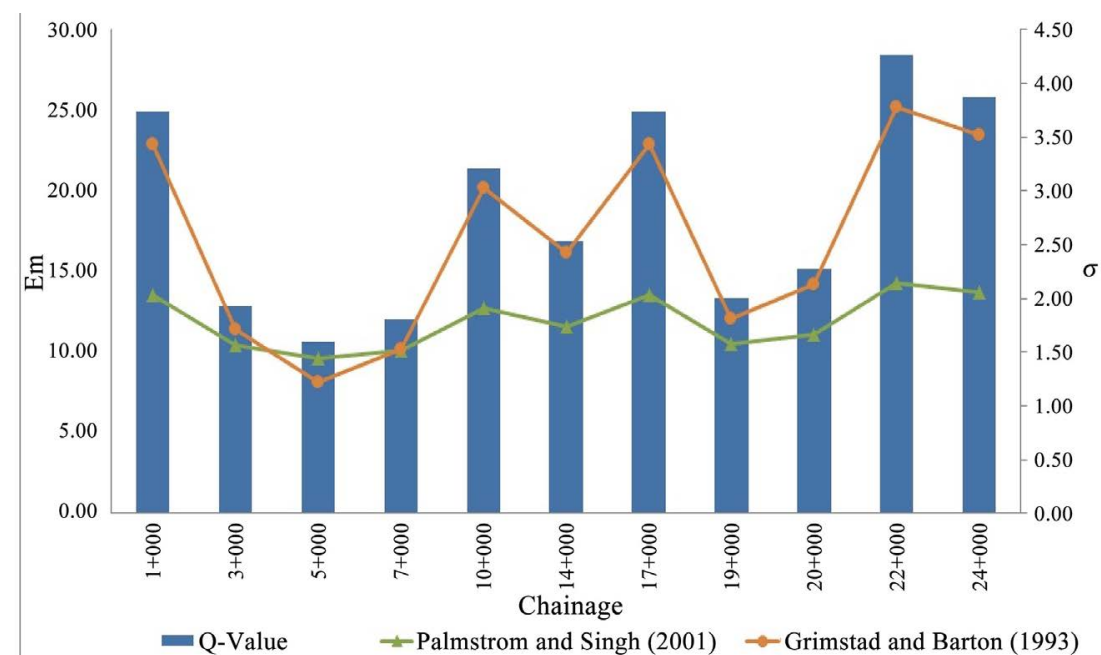

(b)

Figure 6. Comparison of $E_{\mathrm{m}}$ values with Q ratings for (a) left; (b) right tunnel alignment. 


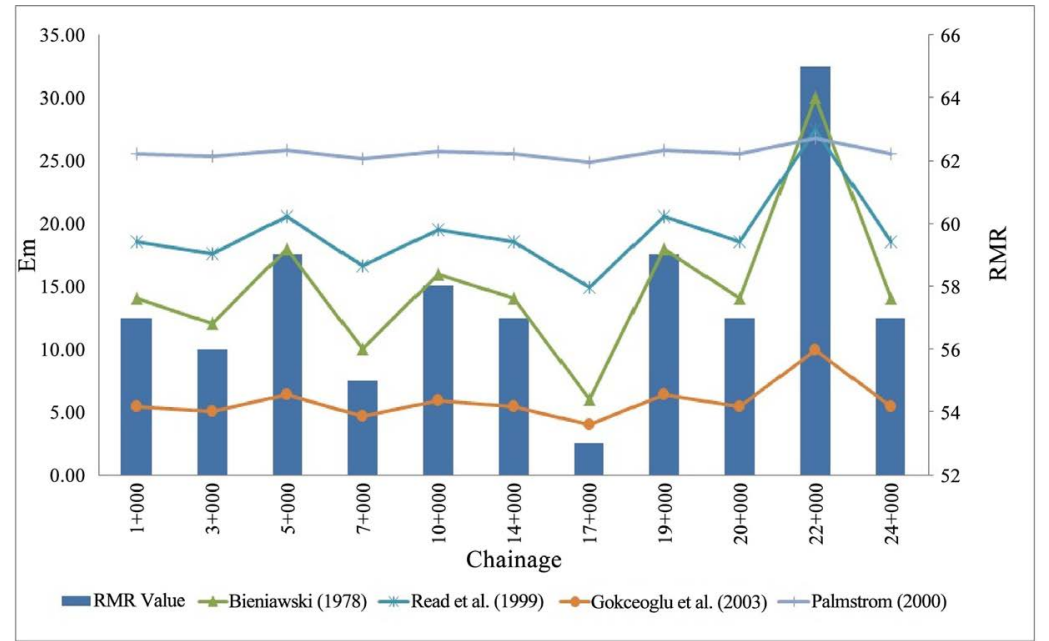

(a)

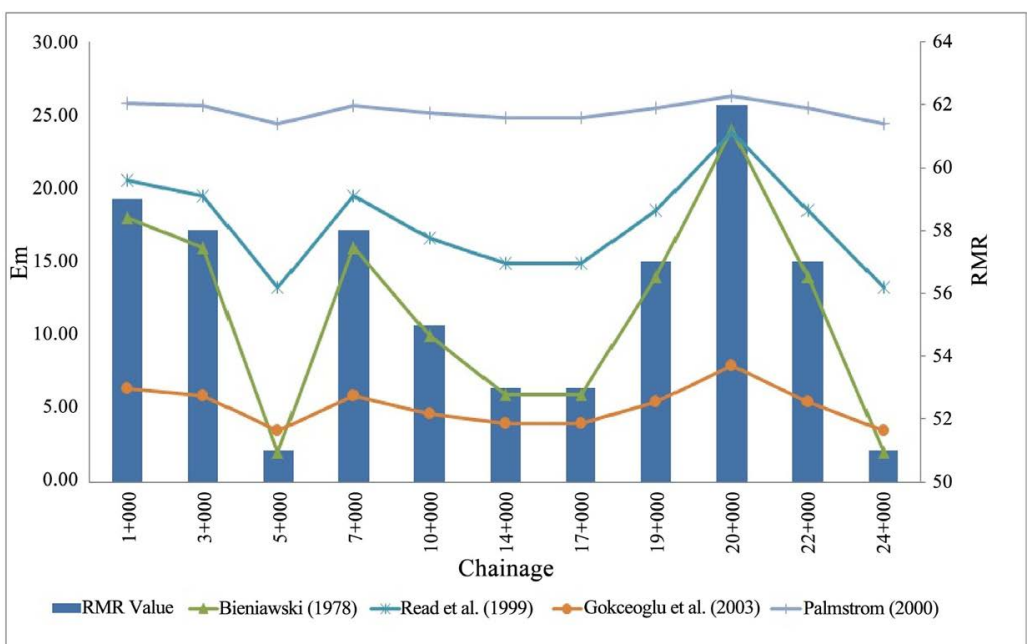

(b)

Figure 7. Comparison of $E_{\mathrm{m}}$ values with Q ratings for (a) left; (b) right tunnel alignment.

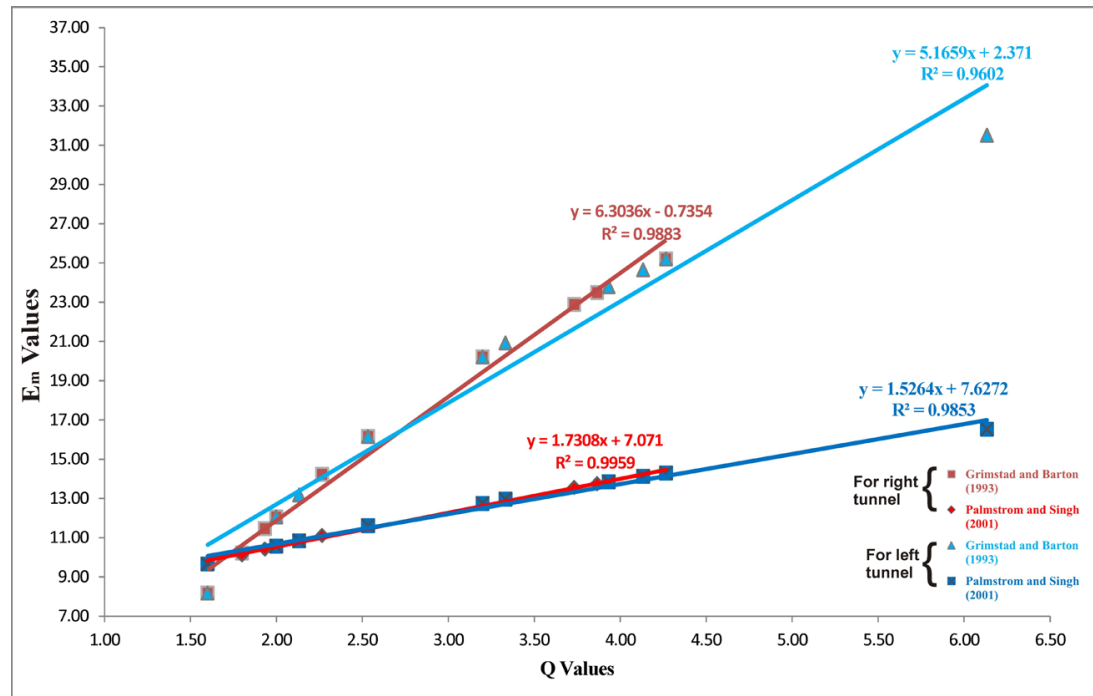

Figure 8. Relationship between estimated $E_{\mathrm{m}}$ values and Q values. 

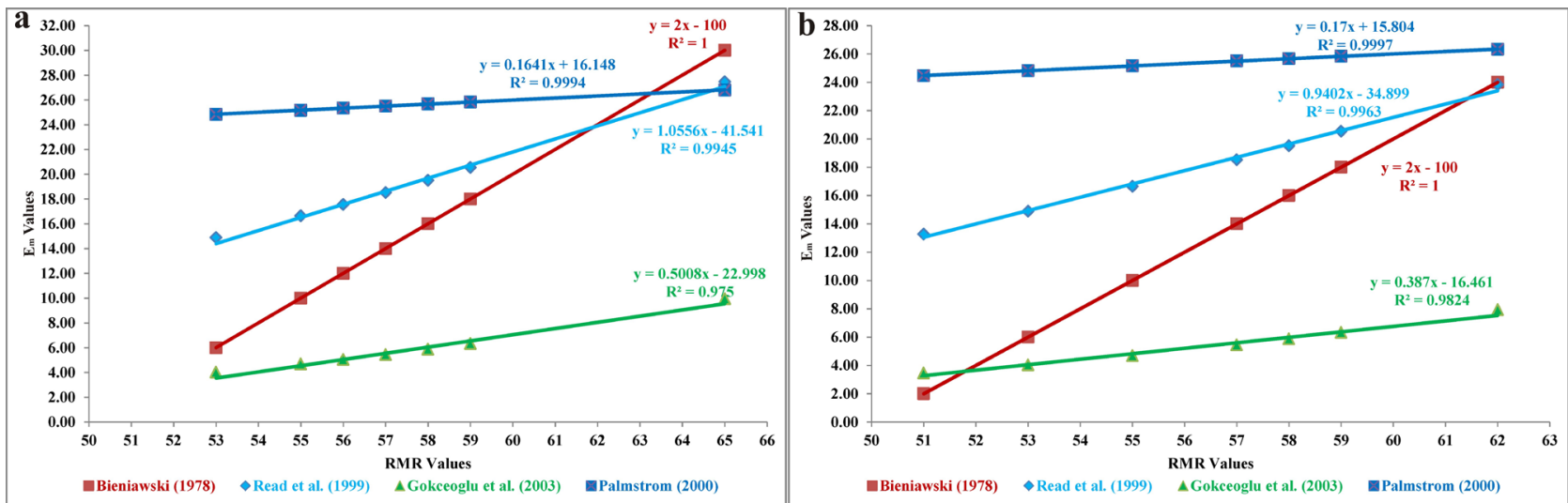

Figure 9. Relationship between estimated $E_{\mathrm{m}}$ values and RMR values for (a) left; (b) right tunnel alignment.
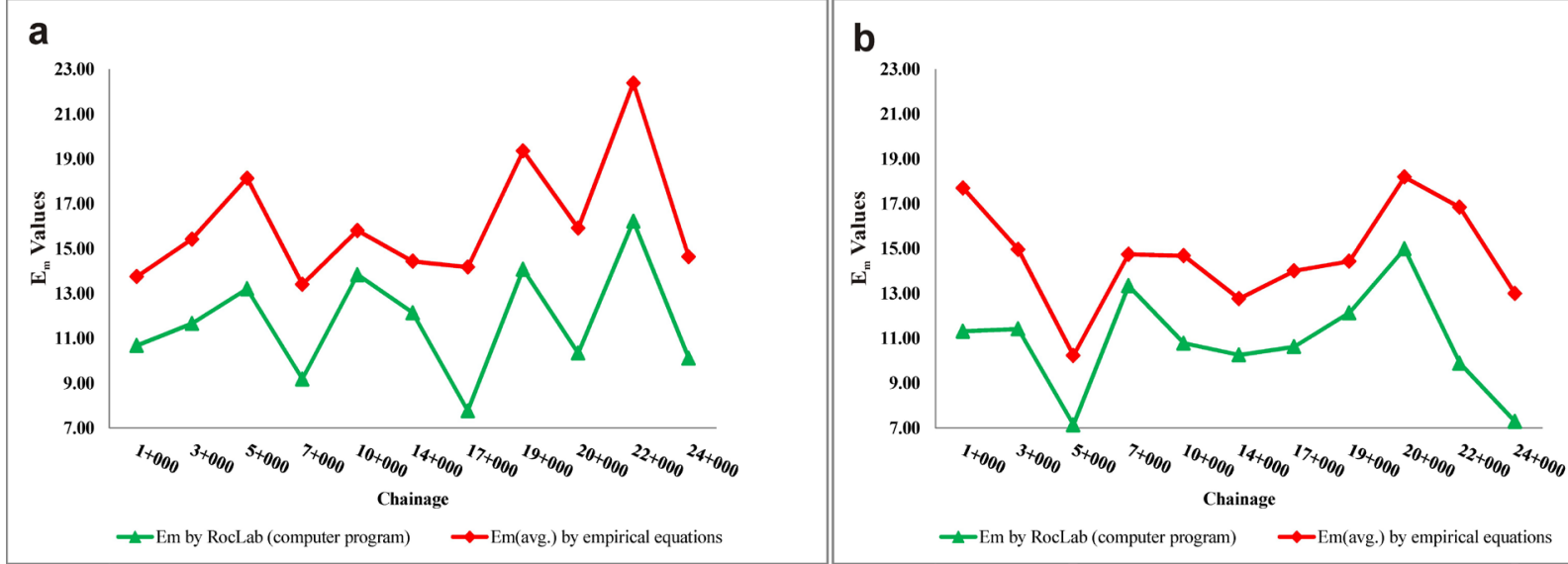

Figure 10. Variation of $E_{\mathrm{m}}$ values obtained by RocLab and empirical equations (avg.) along (a) left; (b) right proposed tunnel alignments.

\section{Conclusions}

Rock mass classification and deformation modulus were studied by RMR and Q schemes on the basis of field studies, laboratory studies, computation work, and graphical representation. RMR yielded fair to good quality rocks with values from 53 to 65 along left tunnel alignment and 51 to 62 along right tunnel alignment. While Q values vary between 1.60 to 6.13 for left tunnel alignment and 1.60 to 4.27 for right tunnel alignment with covering a range of poor to fair quality rocks. This study has predicted that segments of left tunnel alignment have fair rocks except for one segment $(20+000-22+000)$ of good quality rocks, but $\mathrm{Q}$ values for the same segments presented poor rock quality except for three segments $(3+000-5+000,17+000-19+000,20+000-22+000)$ that designated fair rock quality. Similarly, for right tunnel alignment, RMR values revealed fair rocks except for one segment $(12+000-15+000)$ of good quality rocks, but $\mathrm{Q}$ yielded poor rock quality except for one segment $(15+000-20+000)$ of fair rock quality. The estimated values of $E_{\mathrm{m}}$ by various equations have the more or less similar trend of variation with respect to rock quality of study area, respectively. The plots of $E_{\mathrm{m}}$ with RMR and Q indicated significant correlations $\left(R^{2}=0.96\right.$ - 
1). Furthermore, this study revealed that $E_{\mathrm{m}}$ values obtained by RocLab are on the lower side as compare to $E_{\mathrm{m}}$ (Avg.) values obtained by empirical equations and both methods have witnessed their results with the almost similar trend of variation. Moreover, it is recommended that detail investigation of joints, weak zones and laboratory analyses for assessment of appropriate support along tunnel alignments would be necessary.

\section{Conflicts of Interest}

The authors declare no conflicts of interest regarding the publication of this paper.

\section{References}

[1] Ritter, W. (1879) Die Statik der Tunnelgewölbe. Springer, German.

[2] Abu-Zeid, N. and Vuillermin, F. (1997) Geochemical Characterisation of the Outcropping Rock Masses of the Immediate Vicinity of Wadi El-Kaffrein Dam Site (Jordan). Bulletin of the International Association of Engineering Geology, 55, 3.

[3] El-Naqa, A. (2001) Application of RMR Q Geomechanical Classification Systems along the Proposed Mujib Tunnel Route, Central Jordan. Bulletin of Engineering Geology and the Environment, 60, 257-269. https://doi.org/10.1007/s100640100112

[4] Barton, N. (2002) Some New Q-Value Correlations to Assist in Site Characterization and Tunnel Design. International Journal of Rock Mechanics and Mining Sciences, 39, 185-216. https://doi.org/10.1016/S1365-1609(02)00011-4

[5] Choi, S.Y. and Park, H.D. (2002) Comparison among Different Criteria of RMR and Q-System for Rock Mass Classification for Tunneling in Korea. Tunneling and Underground Space Technology, 17, 391-401. https://doi.org/10.1016/S0886-7798(02)00063-9

[6] Ramamurthy, T. (2004) A Geo-Engineering Classification for Rocks and Rock Masses. International Journal of Rock Mechanics and Mining Sciences, 41, 89-101. https://doi.org/10.1016/S1365-1609(03)00078-9

[7] Hoek, E. and Diederichs, M.S. (2006) Empirical Estimation of Rock Mass Modulus. International Journal of Rock Mechanics and Mining Sciences, 43, 203-215. https://doi.org/10.1016/j.ijrmms.2005.06.005

[8] Naithani, A.K., Bhatt, A.K. and Murthy, K.S.K. (2009) Geological and Geotechnical Investigations of Loharinag-Pala Hydroelectric Project, Garhwal Himalaya, Uttarakhand. Journal of the Geological Society of India, 73, 821-836. https://doi.org/10.1007/s12594-009-0066-0

[9] Ozsan, A., Hakan, B., Serdar, Y. and Ozkan, C. (2009) Engineering Geological Evaluation and Preliminary Support Design for the Metro Extension Tunnel, Ankara, Turkey. Bulletin of Engineering Geology and the Environment, 68, 397-408. https://doi.org/10.1007/s10064-009-0192-3

[10] Gupta, M.C., Singh, B.K. and Singh, K.N. (2011) Engineering Geological Rock Mass Classification of Punasa Tunnel Site, Khandwa District, Madhya Pradesh. Journal of the Geological Society of India, 77, 269-272. https://doi.org/10.1007/s12594-011-0034-3

[11] Gurocak, Z. (2011) Analyses of Stability and Support Design for a Diversion Tunnel at the Kapikaya Dam Site, Turkey. Bulletin of Engineering Geology and the Environment, 70, 41-52. https://doi.org/10.1007/s10064-009-0258-2 
[12] Mondal, M.E.A., Siddique, T., Alam, M. and Alam, M.M. (2016) Rock Mass Rating and Kinematic Analysis for Slope Stability Investigation of Utari Dam, Lalitpur District, Uttar Pradesh. Journal Geological Society of India, 87, 463-468. https://doi.org/10.1007/s12594-016-0414-9

[13] Isik, N.S., Ulusay, R. and Doyuran, V. (2008) Deformation Modulus of Heavily Jointed-Sheared and Blocky Greywackes by Pressuremeter Tests: Numerical, Experimental and Empirical Assessments. Engineering Geology, 101, 269-282. https://doi.org/10.1016/j.enggeo.2008.06.004

[14] Ajalloeian, R. and Mohammadi, M. (2014) Estimation of Limestone Rock Mass Deformation Modulus Using Empirical Equations. Bulletin of Engineering Geology and the Environment, 73, 541-550. https://doi.org/10.1007/s10064-013-0530-3

[15] Bieniawski, Z.T. (1978) Determining Rock Mass Deformability: Experience from Case Histories. International Journal of Rock Mechanics and Mining Sciences, 15, 237-247. https://doi.org/10.1016/0148-9062(78)90956-7

[16] Barton, N., Loset, F., Lien, R. and Lunde, J. (1980) Application of the Q-System in Design Decisions Concerning Dimensions and Appropriate Support for Underground Installations. International Conference on Sub-Surface Space, Rock Store, Stockholm, Sweden, 23-27 June 1980, 553-561.

[17] Serafim, J.L. and Pereira, J.P. (1983) Considerations on the Geomechanical Classification of Bieniawski. Proceedings of International Symposium on Engineering Geology and Underground Openings, Lisbon, Portugal, 1983, 1133-1144.

[18] Nicholson, G.A. and Bieniawski, Z.T. (1990) A Nonlinear Deformation Modulus Based on Rock Mass Classification. International Journal of Mining and Geological Engineering, 8, 181-202. https://doi.org/10.1007/BF01554041

[19] Mitri, H.S., Edrissi, R. and Henning, J. (1994) Finite Element Modeling of Cable Bolted Stopes in Hard Rock Ground Mines. Proceedings of SME Annual Conference, Albuquerque, NM, USA, 14-17 February 1994, 94-116.

[20] Read, S.A.L., Richards, L.R. and Perrin, N.D. (1999) Applicability of the Hoek-Brown Failure Criterion to New Zealand Greywacke Rocks. Proceedings of the 9th International Congress on Rock Mechanics, Paris, France, 25-28 August 1999, 655-660.

[21] Palmstrom, A. and Singh, R. (2001) The Deformation Modulus of Rock Masses: Comparisons between in Situ Tests and Indirect Estimates. Tunnelling and Underground Space Technology, 16, 115-131. https://doi.org/10.1016/S0886-7798(01)00038-4

[22] Gokceoglua, C., Sonmeza, H. and Kayabasi, A. (2003) Predicting the Deformation Moduli of Rock Masses. International Journal of Rock Mechanics and Mining Sciences, 40, 701-710. https://doi.org/10.1016/S1365-1609(03)00062-5

[23] Kayabasi, A., Gokceoglu, C. and Ercanoglu, M. (2003) Estimating the Deformation Modulus of Rock Masses: A Comparative Study. International Journal of Rock Mechanics and Mining Sciences, 40, 55-63. https://doi.org/10.1016/S1365-1609(02)00112-0

[24] Sonmez, H., Gokceoglu, C. and Ulusay, R. (2004) Indirect Determination of the Modulus of Deformation of Rock Masses Based on the GSI System. International Journal of Rock Mechanics and Mining Sciences, 1, 849-857. https://doi.org/10.1016/j.ijrmms.2003.01.006

[25] Zhang, L. and Einstein, H.H. (2004) Using RQD to Estimate the Deformation Modulus of Rock Masses. International Journal of Rock Mechanics and Mining Sciences, 41, 337-341. https://doi.org/10.1016/S1365-1609(03)00100-X 
[26] Tahirkheli, R.A.K. and Jan, M.Q. (1979) A Preliminary Geological Map of Kohistan and Adjoining Areas. National Centre of Excellence in Geology, University of Peshawar, Pakistan.

[27] International Society of Rock Mechanics (1978) Suggested Methods for the Quantitative Description of Discontinuities in Rock Masses. International Journal of Rock Mechanics and Mining Sciences, 15, 319-368.

[28] Piteau, D.R. (1973) Characterizing and Extrapolating Rock Joint Properties in Engineering Practice. Rock Mechanics, 2, 5-31.

[29] Bieniawski, Z.T. (1989) Engineering Rock Mass Classifications. Wiley, New York.

[30] Bieniawski, Z.T. (1973) Engineering Classification of Jointed Rock Masses. South African Institution of Civil Engineers, 15, 335-344.

[31] Bieniawski, Z.T. (1974) Geomechanics Classification of Rock Masses and Its Application in Tunneling. 3rd Congress of the International Society of Rock Mechanics, Denever National Academy of Sciences, Washington DC, 1-7 September 1974, 27-32.

[32] Bieniawski, Z.T. (1976) Rock Mass Classification in Rock Engineering. In: Bieniawski, Z.T., Ed., Proceedings of the Symposium on Exploration for Rock Engineering, Balkema, Cape Town, 97-106.

[33] Bieniawski, Z.T. (1979) Thegeomechanics Classification in Rock Engineering Applications. Proceedings of the 4th International Congress Rock Mechanics, Montreux, 2-8 September 1979, 41-48.

[34] Barton, N., Lien, R. and Lunde, J. (1974) Engineering Classification of Rock Masses for the Design of Tunnel Support. Rock Mechanics, 6, 189-236.

https://doi.org/10.1007/BF01239496

[35] Stephens, R.E. and Banks, D.C. (1989) Moduli for Deformation Studies of the Foundation and Abutments of the Portugues Dam-Puerto Rico. In: Rock Mechanics as a Guide for Efficient Utilization of Natural Resources. Proceedings of the 30 th US Symposium, Balkema, Rotterdam, 31-38.

[36] Diederichs, M.S. and Kaiser, P.K. (1999) Stability of Large Excavations in Laminated Hard Rock Masses: The Voussoir Analogue Revisited. International Journal of Rock Mechanics and Mining Sciences, 36, 97-117. https://doi.org/10.1016/S0148-9062(98)00180-6

[37] Carvalho, J. (2004) Estimation of Rock Mass Modulus.

[38] Hoek, E., Carranza-Torres, C.T. and Corkum, B. (2002) Hoek-Brown Failure Criterion-2002 Edition. Proceedings of the Fifth North American Rock Mechanics Symposium, Toronto, 7-10 July 2002, 267-273.

[39] Karaman, K., Ferdi, C. and Ayhan, K. (2015) A Comparative Assessment of Rock Mass Deformation Modulus. International Journal of Mining Science and Technology, 25, 735-740. https://doi.org/10.1016/j.ijmst.2015.07.006

[40] Hamid, R.N., Abdolhadi, G., Seyed, A.M. and Vahab, S. (2014) On the Use of the RMR System for Estimation of Rock Mass Deformation Modulus. Bulletin of Engineering Geology and the Environment, 73, 531-540. https://doi.org/10.1007/s10064-013-0522-3

[41] Grimstad, E. and Barton, N. (1993) Updating the Q-System for NMT, In: Proceedings of the International Symposium on Sprayed Concrete, Norwegian Concrete Association, Oslo, Norway, 20.

[42] Palmstrom, A. (2000) Recent Developments in Rock Support Estimates by the RMi. International Journal of Rock Mechanics and Mining Sciences, 6, 1-19. 
[43] Panthee, S., Singh, P.K., Kainthola, A., et al. (2018) Comparative Study of the Deformation Modulus of Rock Mass. Bulletin of Engineering Geology and the Environment, 77, 751-760. 